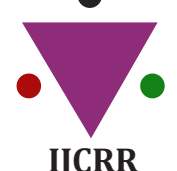

Section: Healthcare

ISI Impact Factor

(2019-20): 1.628

IC Value (2019): 90.81

$\operatorname{SJIF}(2020)=7.893$

(c) (i) (3)

Copyright@IJCRR

\title{
Acid-Base Imbalance and Electrolyte Abnormalities in Acute Alcoholic Intoxication
}

\section{Girish ${ }^{1}$, B Nivedana Lakshmi ${ }^{2}$, Bhat Sonal ${ }^{3}$, Hejamady Ismail Mohammed ${ }^{4}$}

'Associate Professor, Department of Medicine, Kasturba Medical College, Manipal Academy of Higher Education, Mangalore-575001, India; ${ }^{2}$ Junior Resident, Kasturba Medical College, Manipal Academy of Higher Education, Mangalore-575001, India; ${ }^{A}$ Associate Professor, Department of Anaesthesiology, Kasturba Medical College, Manipal Academy of Higher Education, Mangalore-575001, India; ${ }^{4}$ Professor of Medicine, Kasturba Medical College, Manipal Academy of Higher Education, Mangalore-575001, India.

\section{ABSTRACT}

Background: Alcohol intoxication contributes to more than 10 million hospital admissions in a year. It is known to cause impairment in cognition and decline in neurological functions as the blood alcohol levels increase. A series of acid base and electrolyte disturbances have been documented in individuals with alcohol use disorder. Alcoholic ketoacidosis is a common manifestation that is known to occur.

Aims: This study was done to study the pattern of acid base and electrolyte abnormalities that were seen in acute alcoholic intoxication and to establish an association between the blood alcohol levels and the abnormalities in acid base and electrolytes.

Methods: 200 patients in acute alcohol intoxication who satisfy the inclusion criteria were chosen and blood alcohol levels were estimated for them. Blood samples for ABG, Electrolytes were drawn.

Results: Of the electrolytes statistically significant negative association with BAC was seen only with serum bicarbonate. A variety of acid base disturbances were seen the most common being compensated respiratory alkalosis - 56 patients (28\%) followed by metabolic acidosis with partial compensation seen in 46 patients (23\%).

Key Words: Acid, Base, Imbalance, Electrolyte, Acute, Alcoholic, Intoxication

\section{INTRODUCTION}

Alcoholism is a global issue. 5.9\% (3.3 million) of the total deaths globally and $5.1 \%$ of the burden of disease( 139 million DALYs) are primarily due to alcohol consumption. ${ }^{1}$

Acute alcohol intoxication is a clinically harmful condition that usually follows the ingestion of a large amount of alcohol. Alcohol causes harm in three ways- intoxication, toxicity, and alcohol use disorder. Symptoms correspond to the blood alcohol concentration (BAC). In alcohol nontolerant individuals BAC of more than $300 \mathrm{mg} / \mathrm{dl}$ is associated with a higher chance of respiratory depression and cardiorespiratory arrest. BAC levels exceeding $500 \mathrm{mg} /$ dl $(108.5 \mathrm{mmol} / \mathrm{l})$ may cause death. However, the fatal dose varies with nontolerant and tolerant individuals. In nontolerant individuals death has been known to occur at relatively lower BACs $(300 \mathrm{mg} / \mathrm{dl})$. In alcohol-dependent patients who have developed tolerance to alcohol owing to repeated exposure, these effects reduce. This is attrib- uted to compensatory changes occurring in the excitatory neurotransmitters like NMDA (N-methyl-D-aspartate) and inhibitory neurotransmitters like GABA (gammaaminobutyric acid). ${ }^{1,2}$

Acute alcohol intoxication is known to produce various metabolic disturbances like hypoglycaemia, lactic acidosis, hypokalaemia, hypomagnesemia, hypoalbuminemia, hypocalcaemia, and hypophosphatemia., ${ }^{2,3}$ Normal body homeostasis undergoes alterations with alcohol intake both acutely and with chronic alcohol use. Metabolic derangement occurs either as a result of its chemical by-products directly or through alcohol-induced tissue or organ injury.,3,5

There is variation in the pattern of alcohol consumption in India and across the world ${ }^{1}$. There is a paucity of studies that correlate alcoholic intoxication and metabolic derangements, especially in the Indian setup. Our study thus focuses on the prevalence and pattern of acid-base and electrolyte abnormalities in acute alcohol intoxication.

\section{Corresponding Author:}

M Girish, Associate Professor, Department of Medicine, Kasturba Medical College, Manipal Academy of Higher Education, Mangalore-575001, India; Email: girish.m@manipal.edu

ISSN: 2231-2196 (Print)

Received: 21.11 .2020
ISSN: 0975-5241 (Online)

Revised: 05.01 .2021
Accepted: 10.03.2021
Published: 05.07 .2021 


\section{AIMS AND OBJECTIVES}

Our study aimed to study acid-base imbalance and electrolyte abnormalities in acute alcohol-intoxicated individuals and to investigate the association of blood-alcohol levels to disturbances in acid-base balance and electrolyte levels.

\section{METHODS}

This was a cross-sectional study conducted in hospitals associated with KMC (Kasturba Medical College), Mangalore. This was a time-bound study conducted from September 2017 to September 2019. The sample size was 200. The study population was selected using convenient sampling.

\section{Method of study}

After clearance from the institutional ethics committee, KMC Mangalore, the study sample collection was started in September 2017. Informed consent obtained from the patient or patient's relatives. The blood sample was drawn for complete blood counts, renal function tests, liver function tests, $\mathrm{ABG}$, electrolytes after ruling out hypo/ hyperglycemia. A blood sample obtained for estimation of blood alcohol levels. Inclusion criteria being age 18 years or more, Satisfying DSM IV-TR criteria for acute alcoholic intoxication, Blood alcohol concentration $80 \mathrm{mg} / \mathrm{dl}$ All patients who fit the inclusion criteria were enrolled into the study. Informed consent obtained from the patient or patient's relatives. Exclusion criteria included sugar levels at presentation $<70 \mathrm{mg} / \mathrm{dl}$ or $>200 \mathrm{mg} / \mathrm{dl}$, concomitant acute intoxication with other poisons/ psychoactive substances, patients on treatment with drugs causing electrolyte/ acid-base disturbances, pregnant/ lactating women, known diabetics, recent use of alcoholcontaining mouthwash/cologne and patients with a known psychiatric disorder.

Blood alcohol levels were estimated using test kits that were based on the enzymatic method with alcohol dehydrogenase. The NADH produced as a result of the action of ADH on alcohol was measured photometrically based on the principle that the rate of change in absorbance is directly proportional to the ethanol concentration

\section{Analysis}

Collected data were coded and entered into and analyzed using SPSS (statistical package for social sciences - version 17)

Results expressed in terms of descriptive statistics - mean, median and percentages

Categorical variables were presented as numerical data statistical comparison was done using ANOVA. the p-value of less than or equal to 0.05 was considered statistically significant.

\section{RESULTS}

A total of 200 patients were studied. The majority of the patients were $<50$ years of age. Of the 200 patients, 197 were males and 3 were females. Estimated blood alcohol levels were predominantly between 101-200 (62.5\%). Low serum sodium was found in $89(44.5 \%)$ patients

Serum sodium was normal in 110 patients (55\%) and high in 1 patient $(0.5 \%)$.

Serum potassium levels were normal in 47 patients $(71.5 \%)$, low in 143 patients $(23.5 \%)$ and was high in 7 patients $(5 \%)$

Chloride levels were Normal in 126 patients (33.5\%), Hypochloremia was seen in 73 patients $(63 \%)$ and hyperchloremia was seen in $1(0.5 \%)$ of the study population

Bicarbonate levels were normal in 18 patients $(9 \%)$, low in 180 patients $(90 \%)$ and were high in $2(1 \%)$ patients.

Serum calcium level was normal in 164 patients (82\%), hypocalcemia was seen in 36 patients $(18 \%)$ and hypercalcemia was not seen in the study population.

The serum magnesium level was normal in 140 patients(70\%), hypomagnesemia was seen in 46 patients $(23 \%)$ and was hypermagnesemia was seen in 14 patients( $7 \%$ ) of the study population.

Serum phosphorus level was normal in 132 patients (66\%), hypophosphatemia was seen in 25 patients(12.5\%) and hyperphosphatemia was seen in 43 patients(21.5\%) of the study population

Acidosis was seen in $33 \%$ of the patients. Alkalosis was seen in $21 \%$.

$\mathrm{pH}$ was normal in $46 \%$ (92 patients) of the study population. There was a significant negative association between $\mathrm{pH}$ levels and BAC with a p-value $<0.005$ ( Table 1)

\begin{tabular}{|c|c|c|}
\hline Pattern of abnormality & Frequency & Percentage \\
\hline Normal & 11 & $5 \cdot 50 \%$ \\
\hline $\begin{array}{l}\text { Partially compensated metabolic } \\
\text { acidosis }\end{array}$ & 46 & $23.00 \%$ \\
\hline Compensated metabolic acidosis & 15 & $7 \cdot 50 \%$ \\
\hline Acute metabolic acidosis & 15 & $7.50 \%$ \\
\hline $\begin{array}{l}\text { Partially compensated respiratory } \\
\text { alkalosis }\end{array}$ & 31 & $15.50 \%$ \\
\hline $\begin{array}{l}\text { Compensated respiratory alka- } \\
\text { losis }\end{array}$ & 56 & $28.00 \%$ \\
\hline Acute metabolic alkalosis & 1 & $0.50 \%$ \\
\hline Compensated metabolic alkalosis & 2 & $1.00 \%$ \\
\hline Acute respiratory acidosis & 2 & $1.00 \%$ \\
\hline
\end{tabular}


Table 1: (Continued)

\begin{tabular}{lcc} 
Pattern of abnormality & Frequency & Percentage \\
Acute respiratory alkalosis & 8 & $4.00 \%$ \\
Mixed disorder & 13 & $6.50 \%$ \\
\hline
\end{tabular}

The most common being compensated respiratory alkalosis - 56 patients (28\%) followed by metabolic acidosis with partial compensation seen in 46 patients $(23 \%)$ - (Table 2,3)

Table 2: Serum Electrolytes

\begin{tabular}{llcccc} 
Serum Electrolytes & & Sodium & Potassium & Chloride & Bicarbonate \\
BAC $\leq 100$ & Mean & 137.24 & 4.23 & 96.55 & 23.03 \\
& SD & 4.73 & 0.63 & 3.33 & 1.85 \\
BAC 101 - 200 & Mean & 136.05 & 3.90 & 93.70 & 19.13 \\
& SD & 9.41 & 0.71 & 7.75 & 3.56 \\
BAC > 200 & Mean & 134.15 & 3.86 & 93.34 & 12.16 \\
& SD & 6.91 & 0.80 & 10.72 & 4.46 \\
Overall & Mean & 135.66 & 4.01 & 94.64 & 17.532 \\
P value & & 0.087 & & & $<0.011$ \\
One Way ANOVA & & & 0.561 & & $<0001$ \\
\hline
\end{tabular}

Table 3: Serum Electrolytes Cont.....

\begin{tabular}{lcccc} 
& & Calcium & Magnesium & Phosphorus \\
BAC $\leq 100$ & Mean & 8.68 & 1.84 & 3.37 \\
BAC 101 - 200 & Mean & 8.41 & 1.95 & 3.51 \\
BAC > 200 & Mean & 8.08 & 1.92 & 3.21 \\
Overall & Mean & 8.413 & 1.92 & 3.32 \\
Pvalue - One Way ANOVA & & 0.390 & 0.689 & 0.832 \\
\hline
\end{tabular}

Of the electrolytes, a statistically significant negative association with BAC was seen only with serum bicarbonate ( Table 4)

Table 4: Arterial Blood Gas

\begin{tabular}{lccccc} 
Arterial Blood Gases & & Arterial pH & $\mathrm{pCO}_{2}$ & Base Excess & Bicarbonate \\
BAC $\leq 100$ & Mean & 7.4 & 37 & -1.4 & 22.81 \\
BAC 101 - 200 & Mean & 7.39 & 30.65 & -5.83 & 18.4 \\
BAC $>$ 200 & Mean & 7.32 & 26.27 & -12.76 & 12.05 \\
Overall & Mean & 7.37 & 30.13 & -7.24 & 17.149 \\
P value & & 0.138 & $<0.0001$ & $<0.0001$ & $<0.0001$ \\
One Way ANOVA & & & & & \\
\hline
\end{tabular}

Statistically significant association with BAC was seen with pCo2, base excess and bicarbonate

\section{DISCUSSION}

This study was done to assess the pattern of electrolyte and acid-base imbalances occurring in individuals with acute alcohol intoxication. It was conducted in KMC Mangaluru and included a study population of 200 . This study also aimed at establishing an association between the electrolyte and acidbase disturbances and the Blood Alcohol Levels. ${ }^{2,3}$

In a previous study done by Elisaf $\mathrm{M}$ et al. ${ }^{4}$ to study the acidbase and electrolyte abnormalities in alcoholics and to establish the pathogenetic mechanisms involved. $40.5 \%$ had acid-base disturbances. $52 \%$ had electrolyte abnormalities in the study

Another study was done by Adewale et al. ${ }^{3}$, to analyze the consequences of alcohol ingestion on the kidney, fluid, elec- 
trolyte and acid-base abnormalities in both acute and chronic ingestion of alcohol. It was found that alcohol ingestion leads to a multitude of deleterious effects on the kidney. It was noticed that effects like tubular dysfunction to acute renal failure occurred with alcohol ingestion. ${ }^{4}$

In a study done in 2015 by Moses Elisaf and RigasKalaitzidis, ${ }^{5}$ which was carried out for investigating the most commonly occurring metabolic abnormalities in alcoholics and the effect of these disturbances in the patients' morbidity and mortality, it was concluded that the most common electrolyte abnormalities that occurred were hypomagnesaemia with its consequences (hypokalemia and hypocalcemia) and hypophosphatemia.

In a study done by Chandini et al. ${ }^{6}$ serum electrolyte abnormalities in alcohol dependant, patients were studied. Clinical variables of alcohol dependence were correlated with the serum electrolytes in this study. Hyponatraemia, hypochloraemia, hypokalaemia was noted in $46 \%, 24 \%, 67 \%$ of the individuals with alcohol dependence syndrome who participated in the study respectively. The amount of alcohol consumption had a statistically significant correlation with hypokalaemia. However, this study was done on alcohol dependant individuals, unlike our study which was done on intoxicated individuals.

Among the electrolytes, even though hyponatremia, hypernatremia, hypocalcaemia, hypophosphatemia, hyperphosphatemia, hypokalaemia, hyperkalaemia, hypomagnesemia, hypermagnesemia, hypochloraemia, hyperchloremia were seen among the study population, none of these had statistical significance. , $^{4,6}$

A statistically significant electrolyte abnormality that was observed was the low bicarbonate value.

In arterial blood gas analysis, all forms of acid-base abnormalities were observed, the predominant ones being partially compensated metabolic acidosis and compensatory respiratory alkalosis. Statistically, a significant correlation was seen between $\mathrm{BAC}$ levels and $\mathrm{pH}, \mathrm{pCO} 2$, base deficit and bicarbonate

\section{LIMITATIONS}

As it was a cross-sectional study, follow up on the prognosis of the patients based on the severity of acid-base and electrolyte disturbances could not be established

Lactic acidosis was not ruled out as serum lactate values were not measured as a part of the study.

Consumption of other substances was ruled out as per history. But biochemical investigation to rule out the same was not performed.
Some per cent of the study population possibly had a component of chronic liver disease which was not ruled out by questionnaires or imaging modalities.

\section{CONCLUSION}

Acute alcohol intoxication is clinically deleterious manifesting in multiple systems.

Our study shows that electrolyte and acid-base abnormalities have been noted in individuals with acute alcohol intoxication.

The most common acid-base abnormality in our study was compensated respiratory alkalosis followed by metabolic acidosis with partial compensation.

In our study, various electrolyte abnormalities were seen, but only serum bicarbonate levels showed a statistically significant negative association with blood alcohol concentration levels.

Alcoholic ketoacidosis can be corrected and treated if detected. Rapid identification of the serum electrolyte disturbances is of major importance for their appropriate management. Hence it is warranted to check for acid-base imbalance and electrolyte disturbances in intoxicated individuals.

\section{Conflict of Interest: Nil}

\section{Source of Funding: Self}

\section{Author Contribution:}

1. M Girish: Principal investigator

2. B Nivedana Lakshmi: Principal investigator

3. Bhat Sonal: Principal investigator

4. Hejamady Ismail Mohammed: Principal investigator and guide.

\section{REFERENCES}

1. Vladimir P, Dag RG, Jürgen R, Kevin S, Gretchen S. Status report on alcohol and health 2014. Int J Clin Pract. 2014; 1 (1): 51-52.

2. Rosato V, Abenavoli L, Federico A, Masarone M, Persico M. Pharmacotherapy of alcoholic liver disease in clinical practice. Int J Clin Pract. 2016;70(2):119-31.

3. Heidland A, Horl WH, Schaefer RM, Teschner M, Weipert J, Heidbreder E. Role of alcohol in clinical nephrology. Klin Wochenschr 1985; 63: 948-58

4. Elisaf M, Merkouropoulos M, Tsianos EV, Siamopoulos KC. Acid-base and electrolyte abnormalities in alcoholic patients. Miner Electrolyte Metab. 1994; 20: 274-81.

5. Elisaf M, Kalaitzidis R. Metabolic abnormalities in alcoholic patients: focus on acid-base and electrolyte disorders. J Alcohol Drug Depend. 2015; 3: 185.

6. Mathai PJ. Serum Electrolytes Levels in Patients with Alcohol Dependence Syndrome. Int J Clin Pract. 2017; 4 (5):992-997. 